\title{
Prescrição inadequada de medicamentos a idosos portadores de doenças crônicas em um plano de saúde no município de São Paulo, Brasil
}

\author{
Inappropriate medication use in older adults with chronic diseases in a health plan \\ in São Paulo, Brazil
}

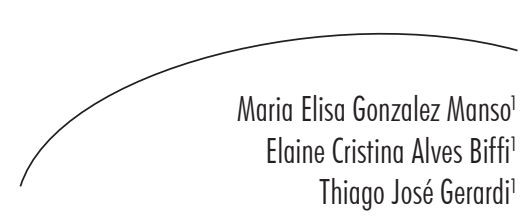

\section{Resumo}

Introdução: A polifarmácia e a prescrição de medicamentos inapropriados para idosos podem causar uma série de efeitos indesejados, comprometendo sua capacidade funcional. Objetivo: Verificação da presença de prescrição de medicamentos inapropriados em um grupo de idosos vinculados a um plano de saúde privado com cobertura na cidade de São Paulo-SP. Metodologia: Estudo realizado mediante a análise de prescrições feitas a um grupo de idosos vinculados a um plano de saúde privado, todos portadores de doenças crônico-degenerativas e acompanhados por médicos de especialidades diversas, na capital do estado de São Paulo, durante os anos de 2012 e 2013. Os medicamentos foram examinados segundo os Critérios de Beers revisados pela American Geriatrics Society em 2012. Resultados: Foram observadas prescrições feitas a 2.500 idosos com um total de 8.760 medicamentos. Destes, 2.926 (33,4\%) foram considerados inapropriados para uso em idosos. Os fármacos mais prescritos foram os anti-inflamatórios não esteroides, seguidos por medicamentos cardiovasculares e de ação central. A maioria desses idosos não possui médico de referência, sendo acompanhados, em média, por quatro médicos de especialidades diferentes. Conclusão: O grupo pesquisado apresenta um número elevado de prescrições de medicamentos inapropriados para uso em idosos, os quais, somados às características próprias, fazem com que esse grupo se torne de alto risco para o aparecimento de iatrogenias. Utilizar critérios de fácil memorização para identificação desses medicamentos e, principalmente, rever a forma como o setor de saúde suplementar brasileiro atende a este segmento etário é de suma importância, a fim de preservar a qualidade de vida desses idosos.

Palavras-chave: Prescrições de medicamentos. Assistência a idosos. Planos de Saúde. 
Abstract

Introduction: Polypharmacy and prescribing inappropriate medication to the elderly can cause a lot of unwanted effects, compromising their functional capacity. Objective: Checking for the presence of inappropriate medication prescription in a group of elderly linked to a private health plan with coverage in the city of São Paulo-SP, Brazil. Methodology: Study based on the analysis of prescriptions made to a group of elderly linked to a private health plan, all bearers of chronic degenerative diseases and accompanied by doctors of various specialities, in the capital of the state of São Paulo, Brazil, during years 2012 and 2013. The medications were examined according to the criteria of Beers reviewed by the American Geriatrics Society in 2012. Results: Were observed prescriptions made to 2,500 elderlies with a total of 8,760 medicines. Of those, 2,926 $(33.4 \%)$ were considered inappropriate for use in older adults. The most prescribed were anti-inflammatory drugs, followed by cardiovascular and centrally acting medications. Most of these aged people have no personal doctor, being accompanied, on average, by 4 doctors of different specialties. Conclusion: The researched group features a high number of prescriptions of inappropriate medications for use in the elderly, which, added to their own characteristics, causes this group to have an increased risk for the appearance of iatrogenics. The use of criteria of easier memorization to identify these drugs and, mainly, to review how the supplementary health sector in Brazil serves this age segment is of paramount importance in order to preserve the quality of life of elderly.
Key words: Drug prescriptions. Elderly health care. Health Plans.

\section{INTRODUÇÃO}

O Brasil e o mundo vêm apresentando um envelhecimento populacional marcante, que pode ser atribuído tanto ao avanço das ciências médicas quanto ao incremento das conquistas sociais (aposentadorias e benefícios) e à melhoria da saúde pública (por exemplo, a cobertura dada pelas imunizações). ${ }^{1}$ Como consequência, os idosos já representam cerca de $12 \%$ da população brasileira. ${ }^{2}$ Paralelamente a esse envelhecimento populacional, observa-se uma mudança do padrão de adoecimento que aflige as populações. Hoje predominam as doenças crônicas, com destaque para as doenças cardiovasculares, principal causa de óbito no mundo. ${ }^{3}$

Estudos populacionais demonstram que, conforme aumenta a idade, aumenta a prevalência das doenças crônicas, destacandose, além das doenças cardiovasculares, os cânceres, as doenças respiratórias e as doenças endócrinas, como o diabetes. ${ }^{3}$ Entre os idosos, as demências, os déficits visuais e auditivos e as doenças osteoarticulares são responsáveis por porção significativa da carga global de doenças
- ou seja, a quantidade de mortes prematuras e incapacidades advindas da presença de uma afecção mórbida. ${ }^{4}$ Não menos de $85 \%$ dos idosos apresentam no mínimo uma doença crônica, e cerca de 10\% apresentam até cinco dessas enfermidades. No entanto, estas não impedem que o idoso possa ter autonomia e realize suas atividades de maneira independente. ${ }^{5}$

A maior prevalência de enfermidades crônicodegenerativas nos idosos culmina, no que diz respeito ao tratamento farmacológico, na prática da polifarmácia, a qual impacta na segurança e qualidade de vida dessas pessoas, tanto por meio do desencadeamento de reações adversas a medicamentos (RAM), quanto mediante prescrição inadequada de medicamentos (PIM). Esta última exacerba não só a incidência de RAM, mas pode ocasionar impactos na capacidade funcional do idoso, pelo aparecimento de interações medicamentosas ou efeitos colaterais indesejados. ${ }^{6-8}$

Embora não haja consenso sobre o número de medicamentos que caracteriza a polifarmácia, esta é definida de forma quantitativa e pode ser considerada quando ocorrer a utilização contínua 
de cinco tipos de fármacos diversos, critério mais prevalente na literatura; ${ }^{9}$ dependendo do tipo de medicamento, no entanto, a utilização de dois fármacos simultaneamente apenas já pode ser um grave problema. ${ }^{10,11} \mathrm{Na}$ gênese da polifarmácia, deve-se ressaltar o número de médicos consultados, a ausência de perguntas ou de informações sobre os medicamentos em uso durante a consulta médica e a automedicação., ${ }^{9,11}$

A terapia farmacológica ainda é um processo de intervenção poderoso para propiciar a melhora do estado de saúde do idoso, desde que racionalmente utilizada.,10 $\mathrm{O}$ uso racional de medicamentos caracteriza-se pela adequação da medicação às necessidades clínicas do paciente, $\mathrm{em}$ doses ajustadas individualmente $\mathrm{e} \mathrm{em}$ regime terapêutico tempo e custo-adequados. ${ }^{10}$ Estima-se que, no Brasil, o uso irracional de medicamentos, caracterizado por polifarmácia, automedicação e interações medicamentosas, constitua um problema de saúde pública, por ocasionar iatrogenia e RAM. ${ }^{10,12}$

Nos idosos, várias alterações fisiológicas decorrentes da própria senescência, como a redução de mecanismos homeostáticos e da função hepática, associados à deficiência visual e declínio cognitivo, podem levar ao aparecimento de RAM. ${ }^{6,13}$ Destacam-se ainda o aumento do tecido adiposo, a perda de massa muscular e de água corporal como elementos que afetam diretamente a ação e duração de muitos fármacos. Com a idade avançada, ocorre redução do clearance renal, causando aumento plasmático dos medicamentos e, em consequência, uma resposta farmacológica maior, elevando o risco de toxicidade. Essas modificações acarretam mudanças na farmacocinética e farmacodinâmica de vários medicamentos. ${ }^{6,8,14}$

As RAMs apresentam, portanto, elevada frequência neste segmento etário, como já dito, e o risco de seu desencadeamento aumenta significativamente quanto maior for a complexidade da farmacoterapia. O risco de RAM com o uso concomitante de dois fármacos é de $13 \%$, valor que chega a $58 \%$ quando se administram cinco medicamentos, e alcança $82 \%$ quando a farmacoterapia chega a sete ou mais., ${ }^{6,13}$ Além do risco de RAM, deve-se ressaltar que quanto maior o numero de fármacos prescritos em conjunto, maior a chance da ocorrência de interação medicamentosa. ${ }^{10}$

A prescrição de fármacos realizada por profissionais habilitados e que apresenta risco significativo de produzir um evento adverso, apesar da existência de alternativas farmacológicas tão ou mais eficazes, é definida como prescrição inadequada de medicamentos (PIM). Esta ocorre quando o risco de aparecimento de efeitos adversos pelo medicamento ultrapassa seus benefícios terapêuticos, quando não há evidências científicas suficientes para seu uso, quando existem alternativas farmacológicas mais seguras disponíveis ou quando o uso do medicamento possa agravar a doença do idoso, e está mais apta a ocorrer quando não é o geriatra que realiza a prescrição. ${ }^{6,7,14} \mathrm{E}$ ainda, o uso de medicamentos por mais tempo do que o necessário, as interações medicamentos-doença e medicamentos-medicamento, bem como a subutilização de fármacos, são consideradas como PIM, ${ }^{8}$ as quais, além de agravarem a saúde do idoso, acarretam elevação dos custos com a atenção a esses agravos..$^{15}$

As alterações de farmacocinética e farmacodinâmica, a prevalência aumentada de doenças crônicas relacionadas à idade e a polifarmácia entre os idosos levaram ao desenvolvimento dos denominados "Critérios de Beers", com o objetivo de facilitar a identificação de PIM nesse segmento etário. ${ }^{14,16}$

Em 1991, após a realização de um painel com especialistas das áreas de Farmacologia e Geriatria, foi publicada uma lista de 30 fármacos que deveriam ser evitados em idosos asilados, independentemente do diagnóstico, dose e frequência de sua utilização. Nessa lista encontravam-se medicamentos como psicofármacos, anti-hipertensivos, hipoglicemiantes orais, anti-inflamatórios não hormonais e analgésicos. Revisada em 1997, a lista passou a incluir idosos com diversos graus de fragilidade e moradores no domicílio. ${ }^{8,14}$ 
Nova revisão ocorreu em 2012, trazendo 53 medicamentos e incluindo a abordagem baseada em evidências científicas. ${ }^{16}$ Os fármacos considerados inapropriados foram incluídos em uma das três categorias: (i) medicamentos e classes de medicamentos potencialmente inapropriados a serem evitados em idosos; (ii) medicamentos potencialmente inapropriados para uso por idosos devido a interações entre medicamento e doença; e (iii) medicamentos que devem ser prescritos com cautela para idosos..$^{14,16}$

A literatura destaca que essas versões dos denominados Critérios de Beers não abrangem todas as principais causas de prescrição potencialmente inapropriada em idosos nem de interações medicamentosas, não contemplando ainda situações tais como subdosagens medicamentosas, uso de fitoterápicos e automedicação. Critica-se a listagem, ainda, por não considerar especificidades de prescrições regionais e incluir somente alguns medicamentos em determinadas classes, destacando-se que os fármacos da lista não são absolutamente contraindicados em idosos. Mesmo assim, por serem de fácil memorização e por sua praticidade, estes critérios são vistos como úteis na prática clínica e têm sido referenciados como um padrão para identificar o uso inadequado de medicamentos neste segmento etário, inclusive em sistemas de saúde privados. Os critérios devem servir como um guia e indicam a necessidade de monitoramento próximo quando algum medicamento da lista for prescrito a um idoso. ${ }^{6-8}$

No Brasil, os idosos são atendidos em suas necessidades de saúde tanto pela rede pública, universal e gratuita, quanto por planos de saúde privados, de acesso limitado à cobertura contratada e à renda do contratante. Este último apresenta composição etária mais envelhecida do que a população brasileira como um todo, sendo que os idosos representam em torno de $11 \%$ do total de beneficiários, podendo atingir até $21 \%$ da carteira em algumas operadoras. ${ }^{17}$

Ao contrário da rede pública brasileira, os planos de saúde privados caracterizam-se por um atendimento fragmentado ao idoso que não considera, na maioria das vezes, suas especificidades. Há poucos médicos geriatras e serviços voltados para a atenção à saúde, sendo frequente a ida a vários médicos especialistas simultaneamente, os quais nem sempre se atêm às características diferenciadas da farmacoterapia dos idosos. Faltam conhecimento teórico e informação sobre modelos de atenção à saúde do idoso que sejam eficientes e adequados. O modelo de atenção, pautado na hiperespecialização e ausência de integralidade na atenção à saúde do idoso predispõe ao uso irracional de medicamentos por este segmento populacional. ${ }^{18}$

Com base no exposto, propôs-se esta pesquisa, que teve como objetivo verificar a presença de prescrição de medicamentos inapropriados em um grupo de idosos vinculados a um plano de saúde privado com cobertura na cidade de São Paulo.

\section{MÉTODOS}

Foi realizado estudo transversal com idosos vinculados a um plano de saúde privado, todos portadores de doenças crônico-degenerativas e acompanhados por médicos de especialidades diversas, na capital do estado de São Paulo, Brasil, durante os anos de 2012 e 2013. Foi analisada a totalidade das medicações prescritas por esses profissionais ao grupo.

Esses idosos, não institucionalizados, participam de um programa de prevenção de complicações de doenças crônicas realizado na operadora de planos de saúde. Trata-se de um programa de adesão espontânea, realizado pelo plano de saúde segundo a metodologia denominada Gerenciamento de Doenças (GD). ${ }^{19}$ Os participantes desse programa possuem diagnóstico de pelo menos uma doença crônica, e alguns já estiveram internados por eventos a elas relacionados, porém todos são independentes nas atividades de vida diária. A execução desse programase dámediantemonitoramentotelefônico alternado com visitas domiciliares, realizados por equipe multiprofissional, cuja frequência varia de acordo com o grau de complexidade de cada idoso assistido. São considerados idosos, para o programa e para o plano de saúde, os indivíduos acima de 60 anos de idade. 
A fim de compor o estudo, optou-se por analisar as prescrições feitas a todos os idosos acompanhados pelo programa nos anos de 2012 e 2013. Para tanto, os idosos foram contatados e informados sobre o objetivo da pesquisa e, caso aceitassem participar, assinaram o Termo de Consentimento Livre e Esclarecido. As prescrições foram lidas e transcritas durante as visitas domiciliares. Dos 2.815 idosos participantes do programa de GD, 2.500 aceitaram participar do estudo.

Os médicos que acompanham esses idosos não têm relação nem interação com o programa, sendo que os idosos os escolhem livremente dentro de uma listagem fornecida pelo próprio plano de saúde. Todos os médicos possuem vínculo empregatício com o convênio médico e atendem nos ambulatórios deste.

A fim de evitar constrangimento ético, a pesquisa foi apresentada tanto à direção do plano de saúde quanto ao diretor clínico responsável pelos ambulatórios, tendo sido aprovada pelas comissões de ética dos ambulatórios pesquisados. Foi solicitado aos pesquisadores que, após o término da pesquisa, os resultados fossem divulgados ao corpo clínico, a fim de permitir a realização de ações de treinamento em serviço, caso fosse necessário. Foi emitida carta de anuência para sua realização e, somente após essa etapa, encaminhada ao Comitê de Ética em Pesquisa da Pontifícia Universidade Católica de São Paulo, constando o plano de saúde como entidade coparticipante, tendo sido aprovada pelo protocolo $\mathrm{n}^{\circ} 219 / 2011$, seguindo as regras da Resolução no 196/96 do Conselho Nacional de Saúde. Somente após esta aprovação, os idosos foram contatados, como anteriormente descrito.

Os dados foram obtidos tanto por meio de um questionário sobre variáveis sociodemográficas, que incluiu perguntas sobre: sexo, idade, estado civil, grau de instrução, doenças diagnosticadas, presença de fatores de risco (dislipidemia ou obesidade, por exemplo), presença de um médico de referência e em quais especialidades médicas os idosos são tratados. As medicações prescritas foram lidas nas receitas médicas prescritas e colhidas durante as visitas domiciliares, as quais foram transcritas pelos pesquisadores e consolidadas em planilha Excel. Os medicamentos foram então classificados de acordo com a Anatomical Therapentic Chemical (ATC), ${ }^{20}$ e segundo os Critérios de Beers revisados pela American Geriatrics Society em 2012, para a prescrição segundo ausência de evidências científicas de utilização. ${ }^{16}$

Apesar de este não ser o objetivo do trabalho, optou-se por apresentar brevemente alguns achados relacionados às interações doençamedicamentos e medicamentos-medicamentos que chamaram a atenção dos pesquisadores por sua frequência e possível impacto na qualidade de vida desses idosos.

\section{RESULTADOS}

Foram analisadas as prescrições realizadas a 2.500 idosos. Estes se encontravam, em sua maioria (1.050-42\%), na faixa etária entre 70 e 75 anos, com um mínimo de 60 anos e um máximo de 101 anos, e média de 71 anos. No grupo, predominaram as mulheres (1.625-65\%).

Sobre o grau de instrução, 68\% (1.700) possuíam, no mínimo, a $5^{\circ}$ série do ensino fundamental completa e a maioria (1.925$77 \%$ ) era aposentada. Dentre as principais doenças e fatores de risco para doenças crônicas encontradas no grupo, destacam-se hipertensão arterial (1.450-58\%), dislipidemia (275-11\%), diabetes (175-7\%) e hipotireoidismo (125-5\%).

Dosidosos pesquisados, 2.225 (89\%) referiram possuir mais de uma doença crônica e/ou fator de risco, sendo que $1.500(60 \%)$ apresentaram quatro ou mais morbidades associadas. As associações mais comumente encontradas foram hipertensão arterial com dislipidemia (575-23\%), hipertensão arterial com diabetes (225-9\%) e diabetes com dislipidemia (75-3\%).

A maioria dos pesquisados não possuía um médico de referência, sendo que o número de médicos que os assistia variou de dois a seis (média de 4), sendo 75\% cardiologistas, reumatologistas e ortopedistas. 
Obteve-se para este grupo um total de 8.760 medicamentos prescritos, com média de 3,5 medicamentos por idoso. Destes fármacos, 2.926 $(33,4 \%)$ foram considerados inapropriados para idosos, segundo os Critérios de Beers. ${ }^{16}$

$\mathrm{Na}$ tabela 1, são apresentados os medicamentos inapropriados prescritos para esse grupo de idosos segundo os Critérios de Beers, ${ }^{16}$ de acordo com o grau de evidência científica. Para tanto, utilizou-se a própria classificação da American Geriatrics Society, independentemente do diagnóstico e condição clínica. Foram incluídos na tabela apenas medicamentos com mais de uma prescrição para melhor visualização. Quando confrontadas as prescrições com as evidências científicas trazidas na última revisão dos Critérios de Beers, todos os medicamentos que aparecem com restrições de uso em idosos por existirem fortes evidências contrárias foram encontrados.

Na tabela 2, são apresentados os principais PIM encontrados nas prescrições feitas a esse grupo de idosos segundo a ATC. Pode-se observar que $1.226(42 \%)$ referem-se aos relacionados ao sistema musculoesquelético, fármacos de ação central, medicamentos cardiovasculares e aqueles utilizados para o sistema digestivo e metabolismo. O ácido acetilsalić́lico foi encontrado na maioria das prescrições, sendo utilizado em doses relacionadas à sua ação trombolítica, mas em 194 prescrições este foi prescrito como anti-inflamatório, daí ter sido, nestes casos, incluído na ATC como relacionado ao sistema musculoesquelético.

Os anti-inflamatórios não hormonais orais não seletivos para inibição da COX foram os mais receitados dentre os medicamentos relacionados ao sistema musculoesquelético (479 prescrições). No grupo dos fármacos cardiovasculares, os antiarrítmicos foram prescritos 119 vezes, destacando-se a amiodarona, com 108 prescrições. Ressaltam-se ainda as prescrições de benzodiazepínicos em 196 casos.

Apesar de não ser objeto deste trabalho, quando analisados os Critérios de Beers em relação às doenças que acometem os idosos, foram encontrados o uso de anti-inflamatórios não hormonais orais não seletivos em pacientes com insuficiência cardíaca (45 casos) e a utilização de benzodiazepínicos em pacientes com histórico de quedas (92 casos).

Foi encontrada, ainda, a prescrição de 66 fórmulas para manipulação, nas quais a principal associação de medicamentos foi a de vários anti-inflamatórios não hormonais orais não seletivos entre si (16 fórmulas) e destes com antidepressivos, como a amitriptilina (10 fórmulas). Foram encontradas ainda também fórmulas com associação de anti-inflamatórios não hormonais orais não seletivos e prednisona, destacando-se que todas estas fórmulas foram prescritas para uso contínuo.

Tabela 1. Distribuição dos medicamentos inapropriados para uso em idosos segundo Critérios de Beers ${ }^{16}$ considerando-se evidências científicas que demonstram que os riscos superam claramente os benefícios. São Paulo-SP, 2012-2013.

\begin{tabular}{lllc}
\hline \multicolumn{2}{c}{ Categoria Terapêutica } & \multicolumn{1}{c}{ Medicamento } & No $^{\circ}$ de Prescrições \\
\hline Anticolinérgicos & Anti-histamínicos de & Dexclorfeniramina & 3 \\
& primeira geração & Difenidramina & 2 \\
& & Hidroxizina & 11 \\
& & Prometazina & 34 \\
& Antiespasmódicos & Escopolamina & 14 \\
\hline Anti-infecciosos & & Nitrofurantoína & 6 \\
\hline
\end{tabular}




\begin{tabular}{|c|c|c|c|}
\hline \multicolumn{2}{|c|}{ Categoria Terapêutica } & Medicamento & $\mathrm{N}^{\mathrm{o}}$ de Prescrições \\
\hline \multirow[t]{9}{*}{ Cardiovasculares } & Bloqueadores alfa-1 & Doxazosina & 40 \\
\hline & \multirow{2}{*}{$\begin{array}{l}\text { Agonista alfa de ação } \\
\text { central }\end{array}$} & Clonidina & 2 \\
\hline & & Metildopa & 13 \\
\hline & \multirow{3}{*}{$\begin{array}{l}\text { Antiarrítmicos (classes } \\
\text { Ia, Ic, III) }\end{array}$} & Amiodarona & 108 \\
\hline & & Quinidina & 2 \\
\hline & & Sotalol & 11 \\
\hline & Digitálico & Digoxina (>0,125 mg/dia) & 21 \\
\hline & $\begin{array}{l}\text { Antagonista dos canais } \\
\text { de Ca (Di-idropiridina) }\end{array}$ & Nifedipina (liberação imediata) & 9 \\
\hline & $\begin{array}{l}\text { Diurético poupador de } \\
\text { potássio }\end{array}$ & Espironolactona (>25 mg/dia) & 62 \\
\hline \multirow[t]{10}{*}{ Ação central } & $\begin{array}{l}\text { Antidepressivos } \\
\text { tricíclicos Terciários } \\
\text { (sozinhos ou em }\end{array}$ & Amitriptilina & 32 \\
\hline & combinação) & Clomipramina & 2 \\
\hline & Barbitúricos & Fenobarbital & 11 \\
\hline & \multirow{6}{*}{$\begin{array}{l}\text { Benzodiazepínicos (de } \\
\text { ação curta, intermediária } \\
\text { ou longa) }\end{array}$} & Alprazolam & 37 \\
\hline & & Estazolam & 4 \\
\hline & & Lorazepam & 12 \\
\hline & & Clonazepam & 65 \\
\hline & & Diazepam & 15 \\
\hline & & Flurazepam & 4 \\
\hline & $\begin{array}{l}\text { Hipnóticos não } \\
\text { diazepínicos }\end{array}$ & Zolpidem & 30 \\
\hline \multirow[t]{3}{*}{ Endócrinos } & & Testosterona & 3 \\
\hline & & $\begin{array}{l}\text { Estrógenos com ou sem } \\
\text { progestágenos }\end{array}$ & 7 \\
\hline & & Insulina (escala móvel) & 159 \\
\hline \multirow[t]{2}{*}{ Gastrointestinal } & & Metoclopramida & 9 \\
\hline & & Óleo mineral oral & 5 \\
\hline \multirow[t]{12}{*}{ Dor } & \multirow{9}{*}{$\begin{array}{l}\text { Anti-inflamatórios não } \\
\text { hormonais orais não } \\
\text { seletivos para inibição da } \\
\text { COX }\end{array}$} & Aspirina $>325 \mathrm{mg} / \mathrm{dia}$ & 284 \\
\hline & & Diclofenaco & 50 \\
\hline & & Ibuprofeno & 11 \\
\hline & & Cetoprofeno & 18 \\
\hline & & Meloxicam & 24 \\
\hline & & Naproxeno & 3 \\
\hline & & Piroxicam & 6 \\
\hline & & Indometacina & 1 \\
\hline & & Ketorolac & 3 \\
\hline & \multirow{3}{*}{$\begin{array}{l}\text { Relaxantes } \\
\text { Musculoesqueléticos }\end{array}$} & Carisoprodol & 38 \\
\hline & & Ciclobenzaprina & 37 \\
\hline & & Orfenadrina & 34 \\
\hline
\end{tabular}


Tabela 2. Distribuição proporcional dos medicamentos inapropriados mais prescritos a um grupo de idosos, segundo a classificação ATC. ${ }^{20}$ São Paulo-SP, 2012-2013.

\begin{tabular}{|c|c|c|}
\hline SISTEMA & $\mathrm{n}$ & $\%$ \\
\hline Cardiovascular & 248 & 8,48 \\
\hline Doxazosina & 30 & \\
\hline Clonidina & 10 & \\
\hline Metildopa & 7 & \\
\hline Amiodarona & 108 & \\
\hline Sotalol & 11 & \\
\hline Digoxina (>0,125 mg/dia) & 21 & \\
\hline Nifedipina (liberação imediata) & 19 & \\
\hline Espironolactona (>25 mg/dia) & 42 & \\
\hline Nervoso Central & 292 & 9,98 \\
\hline Hidroxizina & 11 & \\
\hline Amitriptilina & 32 & \\
\hline Clomipramina & 12 & \\
\hline Fenobarbital & 11 & \\
\hline Alprazolam & 37 & \\
\hline Estazolam & 14 & \\
\hline Lorazepam & 31 & \\
\hline Clonazepam & 75 & \\
\hline Diazepam & 25 & \\
\hline Flurazepam & 14 & \\
\hline Zolpidem & 30 & \\
\hline Musculoesquelético & 479 & 16,37 \\
\hline Diclofenaco & 76 & \\
\hline Ibuprofeno & 21 & \\
\hline Cetoprofeno & 18 & \\
\hline Meloxicam & 44 & \\
\hline Naproxeno & 11 & \\
\hline Piroxicam & 16 & \\
\hline Orfenadrina & 39 & \\
\hline Carisoprodol & 38 & \\
\hline Ciclobenzaprina & 22 & \\
\hline Ácido acetilsalicilico (>325 mg/dia) & 194 & \\
\hline Digestivo e metabolismo & 207 & 7,07 \\
\hline Insulina & 149 & \\
\hline Escopolamina & 14 & \\
\hline Metoclopramida & 29 & \\
\hline Óleo mineral oral & 15 & \\
\hline Principais PIM & 1.226 & 41,90 \\
\hline
\end{tabular}




\section{DISCUSSÃO}

O grupo de idosos participantes da pesquisa não difere quanto aos aspectos sociodemográficos de outros grupos já estudados na saúde suplementar brasileira. Observa-se a predominância de mulheres, com idade acima de 70 anos e elevado grau de instrução..$^{21,22}$

Chamam a atenção a ausência de médico de referência e o número de médicos consultados pelos idosos do grupo, corroborando a já mencionada ausência, por parte do setor de saúde suplementar, de programas de atenção à saúde do idoso. Entretanto, o grupo pesquisado é diferenciado dentro do próprio setor de planos de saúde, pois, como mencionado, fazem parte de um programa para o qual se exige, como critério de inclusão, já ter diagnóstico de uma doença crônica ou ter tido internações por eventos a ela relacionados, o que impõe ao grupo maior vulnerabilidade. Daí a importância de evitar ao máximo a prescrição e o uso de medicamentos inadequados, pela maior probabilidade de aparecimento de RAM.

Vários estudos demonstram que a prescrição de medicamentos inapropriados em idosos está fortemente associada com o desencadeamento de RAM e hospitalizações e, quando associada à presença de comorbidades e polifarmácia, expõe os idosos a um risco elevado de mortalidade. A presença, na prescrição, de pelo menos um medicamento inapropriado, dobra o risco de desencadeamento de RAM, que são responsáveis por aproximadamente $24 \%$ das internações neste segmento etário e constituem a quinta causa de óbito entre os idosos. ${ }^{6,12,14}$

Alguns indivíduos pertencentes ao grupo pesquisado possuem várias morbidades, alguns com quatro ou mais doenças em associação, sendo a média de medicamentos em uso pelo grupo de 3,5 medicamentos por dia, o que torna este grupo de risco para o desencadeamento de RAM na presença do uso de medicamentos inapropriados.
A porcentagem de PIM encontrada nesta pesquisa é elevada, $33,4 \%$, o que pode ser atribuído, em parte, às próprias características do grupo, mas não difere de outros achados na literatura. Esta aponta que, quando aplicados os Critérios de Beers, a prevalência de PIM varia, nos diversos países, entre 27 e $46 \%{ }^{6}{ }^{6,13}$

Praxedes et al. ${ }^{12}$ destacam pesquisas realizadas com idosos acima de 65 anos, em ambiente hospitalar, onde $54,5 \%$ a $67 \%$ das prescrições contêm pelo menos um medicamento inapropriado. Em estudo realizado na Europa ${ }^{23}$ com idosos que recebiam atenção domiciliar nas áreas metropolitanas da República Checa, Dinamarca, Finlândia, Islândia, Itália, Holanda, Noruega e Reino Unido, verificou-se que 19,8\% desses idosos utilizam pelo menos uma medicação inadequada, existindo diferenças significativas entre as prescrições realizadas na Europa Oriental (41,1\% na República Checa) e na Europa Ocidental (média de 15,8\%, variando de 5,8\% na Dinamarca a 26,5\% em Itália). Este estudo encontrou relação entre a PIM e a má situação econômica do paciente, a presença de polifarmácia e de depressão.

No Brasil, estudos mostram que, na cidade de Diamantina-MG, a prevalência de PIM pelos Critérios de Beers foi de 42,32\%, ${ }^{10}$ enquanto que no estudo $\mathrm{SABE},{ }^{14}$ realizado com idosos no município de São Paulo-SP, a prevalência de PIM foi $28 \%$.

Em pesquisa realizada com usuários idosos vinculados a um plano de saúde eque participavam de um programa de gerenciamento de doenças na Suíça ${ }^{24} 22,5 \%$ apresentavam prescrições de medicamentos inadequados, sendo, entretanto, essa taxa menor do que a encontrada para pacientes que não estavam vinculados a nenhum programa semelhante. O grupo de pesquisados descrito neste artigo foi composto por idosos que participavam desses programas, como já mencionado, mas a porcentagem de prescrições de medicamentos inapropriados encontrada está, inclusive, acima da obtida pelo estudo $\mathrm{SABE}^{14}$ para a mesma cidade onde esses idosos residem. 
Como citado, vários estudos demonstram associação significativa para o desencadeamento de RAM em idosos com pelo menos um medicamento inapropriado prescrito, daí a importância de detectar e evitar ao máximo a prescrição desses medicamentos. ${ }^{6,13,24,25}$

No grupo de idosos pesquisado,
os medicamentos inapropriados mais
prescritos foram os relacionados ao sistema musculoesquelético e, dentre estes, os antiinflamatórios não esteroides (AINE) não seletivos, os quais têm grande propensão para causar dispepsia e pirose, além de ulceração gástrica e duodenal. Outro efeito importante são os distúrbios da função plaquetária, com aumento do tempo de sangramento. Quanto à função renal, esses AINEs podem diminuir o fluxo sanguíneo renal e a taxa de filtração glomerular, principalmente em pacientes que já possuem morbidades cardíacas, hepáticas e renais, doenças presentes em vários dos idosos pertencentes ao grupo pesquisado e para os quais estes fármacos foram prescritos. ${ }^{26}$

Os AINEs também promovem a retenção de sal e água, por reduzirem a inibição da reabsorção de cloreto e da ação do hormônio antidiurético, causando edema e descompensação da pressão arterial em pacientes hipertensos controlados, além de poderem levar à hiperpotassemia. ${ }^{26}$ Dos doentes pesquisados, ressalta-se que 58\% apresentam hipertensão, o que torna o grupo sensível ao aparecimento desses problemas.

Estudos demostram que, quando aplicados os Critérios de Beers, os AINEs são drogas frequentemente prescritas, assim como a amitriptilina, os anti-histamínicos e os benzodiazepínicos, estes diretamente relacionados com o desencadeamento de RAM, sobretudo relacionadas a quedas. . $^{6,712,15,27}$

Os benzodiazepínicos têm indicações como drogas ansiolíticas e adjuvantes no tratamento de transtornos psiquiátricos, além de serem relaxantes musculares e anticonvulsivantes. Por serem altamente lipossolúveis, apresentam rápida absorção e distribuição para o sistema nervoso central. Têm metabolização hepática e são classificados de acordo com sua meia vida plasmática, a qual determina seu tempo de ação, bem como pelo grau de afinidade ao receptor benzodiazepínico. ${ }^{12,14,26}$

Independentemente da faixa etária, os benzodiazepínicos podem apresentar como efeitos sonolência diurna, diminuição da coordenação motora, alteração da memória (amnésia anterógrada), tontura, zumbidos, quedas (e, consequentemente, fraturas), reação paradoxal (excitação, agressividade e desinibição, estas mais frequentes em idosos e crianças), além do risco de dependência. ${ }^{14}$ Nos idosos, devido tanto ao aumento do tecido adiposo quanto à diminuição da massa muscular, além de outras alterações fisiológicas, esses medicamentos têm sua farmacocinética e farmacodinâmica alteradas. Assim, podem causar intoxicações e efeitos colaterais com maior facilidade, sendo que a tontura seguida de queda e fratura são eventos de principal importância nessa faixa etária. ${ }^{12}$

Entre o grupo de idosos participantes desta pesquisa, observou-se não só a prescrição de benzodiazepínicos em 196 casos, mas em 92 deles os pacientes já possuíam histórico de quedas, o que torna mais preocupante o quadro, pois o uso deste tipo de medicamento está estatisticamente relacionado a um maior índice de quedas e fraturas nesta faixa etária. ${ }^{28}$ As evidências científicas trazidas pela última revisão dos Critérios de Beers destacam que este tipo de medicamento deve ser evitado para tratamento de insônia em idosos, justificativa para utilização nos 92 casos destacados. ${ }^{16}$

Pesquisas mostram a elevada porcentagem de idosos, principalmente os que se encontram em instituições de longa permanência, que recebem psicotrópicos, sobretudo antidepressivos e neurolépticos. Estas prescrições podem ser atribuídas a vários motivos, que vão desde o alto número de pacientes com problemas psiquiátricos que são recebidos nessas instituições até um padrão de abuso ou dependência de drogas, o qual pode advir do mau uso de determinadas 
medicações antes mesmo da ocorrência da hospitalização. ${ }^{6,25}$

Antidepressivos tricíclicos afetam os neurotransmissores e podem causar, pelo bloqueio dos receptores colinérgicos, retenção urinária, taquicardia e delirium, além de interagir com várias drogas. ${ }^{27} \mathrm{Já}$ os anti-histamínicos de primeira geração, por penetrarem facilmente pela barreira hemato-encefálica e não serem antagonistas seletivos dos receptores histamínicos, possuem ação anticolinérgica, antidopaminérgica e antisserotoninérgica, desencadeando sonolência, sedação, fadiga, redução das funções cognitivas, diminuição da memória e do desempenho psicomotor, o que também pode ser associado a quedas em idosos. ${ }^{28}$

Todos esses medicamentos foram encontrados entre as prescrições realizadas aos idosos participantes da pesquisa, inclusive em medicamentos formulados, bem como medicamentos com ação cardiovascular, em que se destaca o antiarrítmico amiodarona como o mais receitado. Este é um fármaco altamente lipossolúvel que se concentra em vários tecidos e é eliminado de forma lenta. Como já mencionado, fármacos lipossolúveis tendem com mais facilidade a provocar intoxicações em idosos. ${ }^{10} \mathrm{~A}$ amiodarona é ainda um análogo do hormônio da tireoide e possui como efeito adverso importante a fibrose pulmonar advinda do seu uso crônico, além de risco de disfunção hepática, neuropatia periférica, fraqueza muscular proximal e fotossensibilidade. Também é um potente inibidor do metabolismo hepático (CYP3A4 e CYP2C9) e da glicoproteína P, o que pode alterar o metabolismo de outros fármacos. ${ }^{26}$

Existem outros critérios que podem ser utilizados a fim de detectar PIM. Em estudo na Argentina, ${ }^{11}$ utilizaram-se os critérios de Beers, PRISCUS e STOPP, encontrando-se 25,5\%, 31,9\% e 21,3\%, respectivamente, de PIM entre os idosos. Já pesquisa realizada na Índia ${ }^{29}$ com 676 pacientes geriátricos foram usados os critérios de Beers e PRISCUS, observandose PIM em porcentagens de 87,3\% pelo primeiro critério e de $31,06 \%$ pelo segundo. As drogas mais frequentemente prescritas foram metoclopramida (54,3\%), alprazolam (9\%), diazepam (8\%), digoxina $>0,125 \mathrm{mg} / \mathrm{dia}(5 \%) \mathrm{e}$ diclofenaco $(3,7 \%)$, sendo visto o uso de AINEs em pacientes com insuficiência cardíaca e renal, como interação droga-doença mais comumente identificada. Chang et al. ${ }^{30}$ aplicaram seis diferentes critérios para investigação de PIM em idosos em Taiwan e encontraram grande variabilidade de resultados, principalmente relacionados à disponibilidade de medicamentos pelo mercado farmacêutico local.

Apesar de não ser objeto deste trabalho, chamou a atenção o encontro não só de 92 prescrições de benzodiazepínicos a pacientes com histórico prévio de quedas, bem como a indicação de AINEs para idosos com insuficiência cardíaca em 45 casos. Também se destaca a presença de fórmulas de manipulação que associam diversos AINEs entre si ou os associam com amitriptilina ou prednisona.

Faustino et al. ${ }^{27}$ destacam que nem sempre o profissional de saúde que trata o idoso tem acesso a protocolos ou educação continuada, daí podem existir falhas na prescrição. Autores ainda ressaltam que a educação continuada dos médicos, associada ao uso de evidências científicas e de critérios simples como o de Beers, podem diminuir o número de prescrições inadequadas. ${ }^{8,27}$ Segundo Oliveira et al., 8 conhecimento básico da efetividade e segurança dos medicamentos é de suma importância quando da prescrição de medicamentos a idosos, além da importância de também conhecer o próprio processo de envelhecimento.

Em revisão sistemática sobre as prescrições a pacientes idosos realizadas na atenção primária à saúde, ${ }^{31}$ notou-se que uma em cada cinco eram prescrições de medicamentos inadequados. Destes, os mais prescritos e com alto risco de desencadear efeitos adversos foram a difenidramina e a amitriptilina, seguidas pelo propoxifeno e doxazoxina - estes, porém, de baixo risco para RAM. Os autores sugerem que, 
além do treinamento dos médicos, é necessário o apoio informatizado a decisão clínica e um prontuário único, a fim de evitar as PIMs.

Esta pesquisa possui algumas limitações. Destaca-se que este trabalho apenas analisou as prescrições realizadas por médicos, não considerando a presença de automedicação, nem avaliou a presença de RAM ou hospitalizações destas decorrentes, tendo-se fixado nas prescrições de medicamentos inadequados segundo os Critérios de Beers, no que tange à presença de evidência científica para o uso da medicação prescrita.

Ressalta-se ainda que se trata de um grupo específico de idosos atendidos por uma operadora de planos de saúde e participantes de um programa de GD, como já citado, o que limita a generalização dos achados. Trata-se de um grupo com várias comorbidades e que é atendido em um modelo assistencial, como apresentado, o que dificulta a integralidade da atenção à saúde do grupo.

\section{CONCLUSÃO}

As interações medicamentosas, a polifarmácia e a automedicação caracterizam o uso irracional de medicamentos, o qual se associa a elevada incidência de RAM, hospitalizações e até óbitos, principalmente entre os idosos. Nestes, as alterações fisiológicas que modificam a farmacocinética e a farmacodinâmica de vários medicamentos potencializam o desencadeamento de reações adversas.

O grupo participante desta pesquisa possui características que os torna vulneráveis a apresentar reações adversas decorrentes de PIM, tais como idade avançada e presença de várias comorbidades. Além dos efeitos advindos do uso isolado de cada fármaco, deve-se ressaltar que esse grupo utiliza, em média, 3,5 fármacos, o que potencializa ainda mais o risco de RAM, como ressalta a literatura.
Para esse grupo, foram encontradas 33,4\% de PIM em 8.760 prescrições analisadas durante os anos de 2012 e 2013, na cidade de São Paulo. Dos medicamentos inapropriados encontrados, destacam-se os relacionados ao sistema musculoesquelético, aos fármacos de ação central, aos medicamentos cardiovasculares e para $\mathrm{O}$ sistema digestivo e metabolismo, compondo $42 \%$, aproximadamente, do total de PIM. As drogas mais prescritas foram os AINEs, seguindo-se os benzodiazepínicos e antiarrítmicos. Foram encontradas, ainda, prescrições de AINEs para pacientes com insuficiência cardíaca e benzodiazepínicos para idosos com histórico de quedas anteriores, bem como formulações magistrais contendo associação de AINEs entre si, com prednisona e amitriptilina.

Deve-se ter cautela ao prescrever medicamentos aos idosos, para que não haja malefícios. Além de utilizar critérios de fácil visualização e memorização, como os Critérios de Beers, a prescrição deve seguir as melhores evidências científicas existentes, adequando-se ao caso concreto. Infelizmente, esta precaução nem sempre é tomada, fato que pode comprometer a qualidade de vida dos idosos, gerando não apenas reações adversas aos medicamentos, mas comprometendo sua capacidade funcional.

Devido ao próprio modelo de atenção preconizado pelo sistema de saúde suplementar brasileiro, há poucos profissionais com adequada formação para a atenção à saúde do idoso e suas especificidades, o que pode levar a uma maior prevalência de PIM, por desconhecimento das alterações farmacocinéticas e farmacodinâmicas relacionadas à senescência.

Acredita-se que esta pesquisa atingiu seu objetivo ao realizar um levantamento sobre as prescrições feitas em ambulatórios próprios de um plano de saúde na cidade de São Paulo-SP, fornecendo subsídios para melhorar a atenção a este segmento etário, que cresce cada dia mais no Brasil. 


\section{REFERÊNCIAS}

1. Olshansky SJ, Beard JR, Börsch-Supan. The longevity dividend: health as an investment. In: Bread J, Biggs S, Bloom D, Fried L, Hogan P, Kalache A, et al. Global population ageing: peril or promise? [Internet]. Geneva: World Economic Forum; 2012 [acesso em 24 set 2012]. Disponível em: http://www. hsph.harvard.edu/pgda/working.htm.

2. Instituto Brasileiro de Geografia e Estatística [Internet]. Rio de Janeiro: IBGE; [1995]- . Sala de Imprensa: IBGE divulga Indicadores Demográficos e de Saúde; 2009 [acesso em 09 abr 2013]; [aproximadamente 1 tela]. Disponível em: http:// saladeimprensa.ibge.gov.br/noticias? view $=$ noticia\&id $=1 \&$ busca $=1 \&$ idnoticia $=1445$.

3. Bloom DE, Cafiero ET, Jané-Llopis E, AbrahamsGessel S, Bloom LR, Fathima S, et al. The Global Economic Burden of Non-Communicable Diseases. Geneva: World Economic Forum; 2011. (PGDA Working Paper no 87)

4. Goulart FAA. Doenças crônicas não transmissíveis: estratégias de controle e desafios para os sistemas de saúde. Kemper ES, revisora técnica. Brasília: OPAS, 2011.

5. Alves LA, Leite IC, Machado CJ. Conceituando e mensurando a incapacidade funcional da população idosa: uma revisão de literatura. Ciênc Saúde Coletiva 2008;13(4):1199-207.

6. Varallo FR, Ambiel ISS, Nanci LO, Galduróz JCF, Mastroianni PC. Assessment of pharmacotherapeutic safety of medical prescriptions for elderly residents in a long-term care facility. Braz J Pharm Sci 2012;48(3):477-85.

7. Farfel JM, Accorsi TAD, Franken M, Doudement SP, Moran M, Iervolino M, et al. Adverse drug events leading to emergency department visits in elderly: the role of inappropriate prescription. Einstein 2010;8(2):175-9.

8. Oliveira MG, Amorim WW, Rodrigues VA, Passos LC. Access of Brazilian elders to potentially inappropriate medications. Rev APS 2011;14(3):258-65.

9. Cabrera M. Polifarmácia e adequação do uso de medicamentos. In: Freitas EV, Py L, editores. Tratado de Geriatria e Gerontologia. Rio de Janeiro: Guanabara-Koogan; 2011. p. 1055-9.

10. Pinto MCX, Ferré F, Pinheiro MLP. Potentially inappropriate medication use in a city of Southeast Brazil. Braz J Pharm Sci 2012;48(1):79-86.
11. Regueiro M, Mendy N, Cañás M. Uso de medicamentos en adultos mayores no institucionalizados. Rev Peru Med Exp Salud Publica 2011;28(4):643-47.

12. Praxedes MFS, Telles PCP Filho, Pinheiro MLP. Identificação e análise de prescrições de medicamentos potencialmente inapropriados para idosos em uma Instituição Hospitalar. Cienc Cuid Saúde 2011;10(2):338-44.

13. Varallo FR, Capucho HC, Planeta CS, Mastroianni PC. Safety Assessment of Potentially Inappropriate Medications (PIM) use in older people and the factors associated with hospital admission. J Pharm Sci 2011;14(2): 283-90.

14. Cassoni TCJ, Corona LP, Romano-Lieber NS, Secoli SR, Duarte YAO, Lebrão ML. Uso de medicamentos potencialmente inapropriados por idosos do Município de São Paulo, Brasil: Estudo SABE. Cad Saúde Pública 2014;30(8):1708-20.

15. Akazawa M, Imai H, Igarashi A, Tsutani K. Potentially inappropriate medication use in elderly Japanese patients. Am J Geriatr Pharmacother. 2010;8(2):146-60.

16. American Geriatrics Society. The American Geriatrics Society Beers update criteria for potentially inappropriate medication use in older adults. J Am Geriatr Soc 2012;60(4):616-631.

17. Leite F. Envelhecimento populacional e a composição etária de beneficiários de planos de saúde. São Paulo: IESS; 2011.

18. Brasil. Agência Nacional de Saúde Suplementar. Plano de cuidado para idosos na saúde suplementar. Rio de Janeiro: ANS; 2012.

19. Sidorov JE. Gerenciamento de doenças: passado, presente e futuro. J Bras Econ Saúde 2012;4:20-26.

20. WHO Collaborating Centre for Drugs Statistics Methodology [Internet]. Oslo: World Health Organization; 2014. Anatomical Therapeutic Chemical (ATC) classification index with defined daily doses (DDDs); 2014 [acesso em 01 dez 2014]; [aproximadamente 1 tela]. Disponível em htpp:// www.whocc.no/atc_ddd_index.

21. Veras RP, Caldas CP, Araújo DV, Kuschnir R, Mendes W. Características demográficas dos idosos vinculados ao sistema suplementar de saúde no Brasil. Rev Saúde Pública 2008;42(3):497-502. 
22. Manso MEG, Ribeiro MP. Caracterização das condições de saúde de um grupo de idosos pertencente a um plano de saúde. Rev. Bras. Med. 201269 (3): 45- 55.

23. Fialová D, Topinková E, Gambassi G, FinneSoveri H, Jónsson PV, Carpenter I, et al. Potentially inappropriate medication use among elderly home care patients in Europe. JAMA 2005;293(11):1348-58.

24. Reich O, Rosemann T, Rapold R, Blozik E, Senn O. Potentially inappropriate medication use in older patients in Swiss Managed Care Plans: prevalence, determinants and association with hospitalization. PLos ONE [Internet] 2014 [acesso em 20 jul 2014];9(8):105425. Disponível em: http://www.plosone.org/article/ info $\% 3$ Adoi $\% 2 F 10.1371 \% 2 F j o u r n a l . p o n e .010542$.

25. Locatelli J, Lira AR, Torraga LK, Paes AT. Inappropriate medications using the Beers criteria in Brazilian hospitalized elderly patients. Consult Pharm 2010; 25(1):36-40.

26. Gilman AG. As bases farmacológicas da terapêutica. Rio de Janeiro: McGraw-Hill; 2005.
27. Faustino CG, Martins MA, Jacob-Filho W. Potentially inappropriate medication prescribed to elderly outpatients at a general medicine unit. Einstein 2011;9(1):18-23.

28. Hamra A, Ribeiro MB, Miguel OF. Correlação entre fratura por queda em idosos e uso prévio de medicamentos. Acta Ortop Bras 2007;15(3):143-5.

29. Jhaveri BN, Patel TK, Barvaliya MJ, Tripathi C. Utilization of potentially inappropriate medications in elderly patients in a tertiary care teaching hospital in India. Perspect Clin Res 2014;5(4):184-9.

30. Chang CB, Chen JH, Wen CJ, Kuo Hsu, Lu IS, ShuChiu L, et al. Potentially inappropriate medications in geriatric outpatients with polypharmacy: application of six sets of published explicit criteria. Br J Clin Pharmacol 2011;72(3):482-9.

31. Opondo D, Eslami S, Visscher S, De Rooij SE, Verheij R, Korevaar JC, et al. Inappropriateness of medication prescriptions to elderly patients in the primary care setting: a systematic review. PLoS ONE 2012;7(8): e43617. 